Abstracts

Abstract Number

Presenter

Exposure

Health domains

Type of research

\section{P-2-08-16}

Luca Del Buono, Andrea Spinazzè*, Davide Campagnolo, Andrea Cattaneo, Paolo Carrer, Domenico Maria Cavallo

ambient air pollution

no health domain

others

\title{
Indoor Air Quality in Bank Offices
}

Background Since the general population spends most of its time into indoor environments, the study of indoor air quality (IAQ) is of great concern for assessing the impact of indoor air pollutants on human health. Aims In this study 6 Italian bank branches, identified as "of concern" on the basis of an "IAQ-perception" questionnaire, were investigated through the measurement of PM10, PM2.5, PM1, PM0.5, PM0.25, HCHO, NO2, CO2, ACH. In order to perform a more detailed study, some other chemicals (aldheydes, terpenes, ozone) were measured in 4 of the selected bank branches. Methods The above mentioned pollutants were twice measured (in summer and winter) for 24 hours through active sampling (PM), passive sampling (VOCs, aldehydes and terpenes) or direct-reading instruments (CO2 and ozone). In each bank branch, sampling was carried out in 2 indoor offices ("teller area" and "consulting area") and 1 outdoor location. A specific check-list has been completed, to collect also information about office construction and design, building services and local environment. Results The analysis (Paired T-Test, One-way-ANOVA, PCA) of collected data showed statistically significant differences by sampling positions, sampling sessions (summer/winter). The highest measured mean concentrations were generally observed in the teller area and during the winter sampling session. Other office characteristics (e.g. relevant refurbishment of the filial), appeared to be important in determining the indoor concentrations of some air contaminants (e.g. HCHO) Conclusions Differences in indoor air quality were observed within and between bank branches. Air pollutant concentrations were also related to spatial and seasonal variations, as well as to some specific building characteristics.

\section{Abstract Number}

Presenter

\section{Exposure}

Health domains

Type of research

\section{P-2-08-17}

Kanistha Chatterjee*, Eric Kettleson, Reshmi Indugula, Patrick Ryan, Sergey Grinshpun, Gary Adamkiewicz, Tiina Reponen

household air pollution

no health domain

exposure measurement

\section{Indoor Air Quality in Green vs. Non-green Homes}

Background: Green building features are designed to have a minimal negative impact on the environment and reduce energy consumption. However, the indoor air quality (IAQ) within green buildings has not yet been quantified. We assessed the indoor air quality in 20 green-renovated and 20 control apartments in Cincinnati, Ohio, USA. A 5-day IAQ assessment in each home included measurement of temperature, relative humidity and ventilation as well as concentrations of fine particles, allergens, volatile organic compounds (VOCs), and pesticides. Aims: Determine how green renovation features affect the indoor air qualityMethods: Temperature and relative humidity were monitored using continuous data loggers. Sampling for particle mass $\leq 2.5 \mu \mathrm{m}$ (PM2.5) was conducted using single-stage Personal Modular Impactors with 37-mm, 2.0- $\mu \mathrm{m}$ pore-size PTFE membrane filters. VOC sampling was performed using passive diffusion dosimeters and pesticide samples were collected from kitchen floor by wiping. Dust samples were vacuumed from beds and kitchen floors and analyzed for allergens. Results: Preliminary data obtained immediately after renovation shows that relative humidity and formaldehyde levels were significantly higher in green vs. non-green homes ( $p=0.04)$. In contrast, isopropyl alcohol levels were significantly higher in non-green homes ( $p=0.05)$. Overall, there were higher detectable levels of pesticides in non-green vs. green homes. Additionally, we identified a trend of higher PM2.5levels in green homes $(p=0.08)$. Higher concentrations of allergens were observed in non-green homes though the difference was not statistically significant.Conclusions: The baseline data immediately after renovation shows conflicting trends between green-renovated and renovated homes for different indoor pollutants. Follow-up sampling performed 6 and 12 months after renovation may help identify how the IAQ will change in green-renovated homes over time. 